\title{
CURRENT-VOLTAGE CHARACTERISTIC OF SEMI-INSULATING GaAs WITH TRAP-FILLING EFFECT
}

\author{
K. KARPIŃSKA AND J. LUSAKOWSKI \\ Institute of Experimental Physics, Warsaw University, Hoża 69, 00-681 Warsaw, Poland
}

(Received August 8, 1990)

\begin{abstract}
A detailed investigation of current-voltage ( $I-V)$ characteristics of semi-insulating GaAs sample was performed in the vicinity of room temperature. The sample with $300 \mathrm{~K}$ resistivity of $2 \times 10^{7} \Omega \mathrm{cm}$ was supplied with guard-ring electrodes which allowed the elimination of surface currents. The observed characteristics started with an ohmic part which was followed by a superlinear current on voltage dependence. At a threshold voltage $V_{\text {th }}$ which corresponds to the electric field of about $2 \mathrm{kV} / \mathrm{cm}$ the current increased abruptly by a few orders of magnitude. The value of $V_{\mathrm{th}}$ increased with the temperature. It is proposed that the observed shape of the $I-V$ curve is caused by the filling of the EL2 level with injected electrons heated by the electric field.
\end{abstract}

PACS numbers: $72.20 . J v$

\section{Introduction}

Since any sample has a non-zero capacitance it collects charge when the current passes through. The injected charge can strongly influence the shape of the current-voltage characteristic [1]. The injected charge is distributed among localized and mobile states. When the injection level is high enough the additional non-equilibrial charge in the conduction band causes stronger than linear current on voltage dependence. What is more, when the injected charge is large enough to fill a set of traps then one observes an abrupt increase of the current. This results from the fact that the injected carriers can only then enter the conduction band. These effects are particularly obvious in high-resistivity materials where the concentration of the injected charge can easily attain the value of a low concentration of thermally generated, free carriers and where the thermal concentration is many orders of magnitude smaller than the concentration of unoccupied trap levels. 


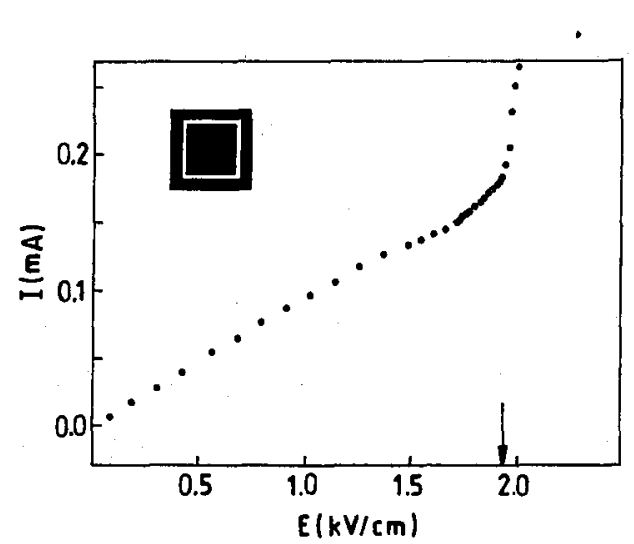

Fig. 1. A typical shape of the current-voltage characteristic. The arrow indicates the threshold electric field. The inset shows the configuration of the electrodes (the shaded area) prepared on both sides of the sample. The edge of the sample is $10 \mathrm{~mm}$ long. The width of the guard-ring and the channel is $1 \mathrm{~mm}$. The sample thickness is $0.035 \mathrm{~cm}$. Note that the areas of the central and the guard-ring electrodes are equal.

Semi-insulating GaAs (SI GaAs) is one of many [2] semiconductors where the effects of injection and trapping can be observed [3]. In this paper we present the results of measurements of $I-V$ characteristics carried out on this material. We suggest that an abrupt increase of the current in the electric field of about $2 \mathrm{kV} / \mathrm{cm}$ is caused by filling the EL2 level with injected electrons which were strongly heated by the electric field.

\section{Experiment}

The sample under investigation was a square plate of SI GaAs with $\mathrm{Au} / \mathrm{Ge} / \mathrm{Ni}$ contacts. Centrally placed square electrodes were surrounded by guard-ring electrodes (see the inset of Fig. 1). This allowed the elimination of surface currents. The sample was placed in a metallic cylinder. The temperature inside the cylinder was automatically controlled and stabilized with an accuracy of $0.1 \mathrm{~K}$. It was measured by a copper-constantan thermocouple. The measurements were done in the temperature range of $298-323 \mathrm{~K}$. The electrical arrangement was as that described in [3]. The values of fitted energies and their errors come from a least square procedure.

\section{Results}

A typical $I-V$ characteristic is shown in Fig. 1. The value of the electric field was obtained by dividing the value of the voltage applied to the sample by the sample thickness. For electric fields up to about $1.7 \mathrm{kV} / \mathrm{cm}$ we observe 

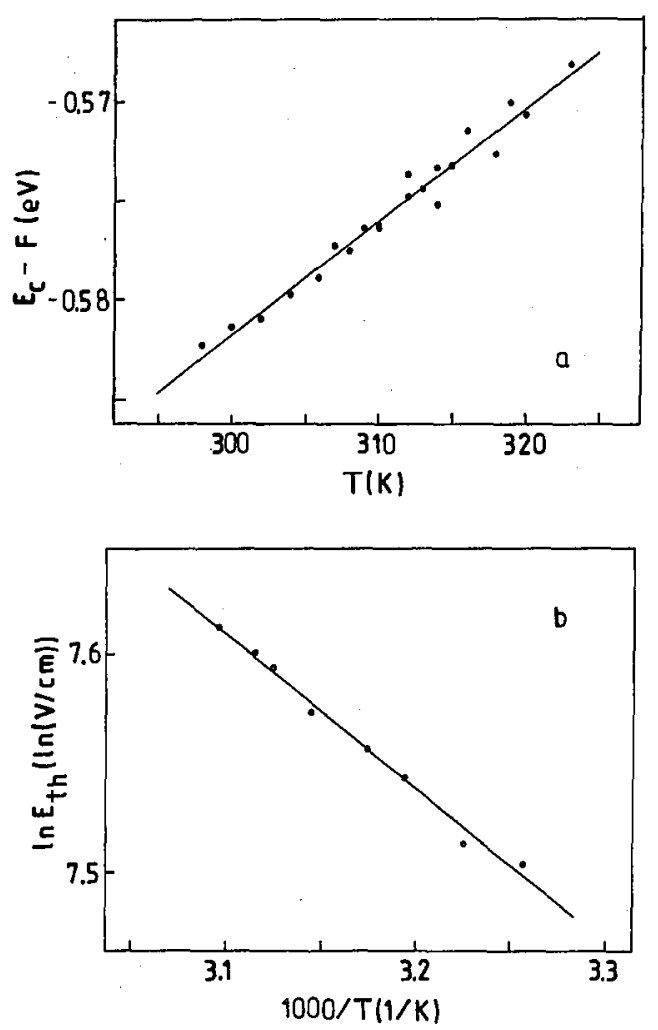

Fig. 2. a) The position of the Fermi level below the bottom of the conduction band as a function of temperature; b) the activation plot of $\ln \left(E_{\mathrm{th}}\right)$ as a function of $1000 / T$.

ohmic behaviour. Above this field there is a transition region where the current is proportional to $V^{\alpha}$ where the exponent $\alpha$ is about 1.6 and is independent of the temperature. The $V^{\alpha}$ dependence ends with an abrupt increase of the current in the electric field $E_{\mathrm{th}}$. The value of $E_{\mathrm{th}}$ increases with the temperature following $\exp (\delta E / k T)$ dependence where $\delta E$ is $(0.060 \pm 0.003) \mathrm{eV}$.

The guard-ring electrodes allowed the investigation of the planar current flow between the central electrodes. In this case the simple formula $R=l / \sigma S$ (where the symbols have their usual meaning) can be applied provided the resistance of the contacts is neglected. From the above formula we found the conductivity of the investigated material equal to $5 \times 10^{-8} \Omega^{-1} \mathrm{~cm}^{-1}$. Basing on the known relations $\sigma=n e \mu$ and $n=N_{\mathrm{C}} \exp \left[-\left(E_{\mathrm{C}}-F\right) / k T\right]$, with the conduction-band density of states $N_{\mathrm{C}}=2\left(2 \pi m k / h^{2}\right)^{3 / 2} T^{3 / 2}$, we calculated the position of the Fermi level $F$ below the bottom of the conduction band $E_{\mathrm{C}}$ as a function of temperature. In the calculation we assumed the electron mobility $\mu=4000 \mathrm{~cm}^{2} / \mathrm{Vs}$ and the effective electron mass $m^{*}=0.07$ of the free electron mass. We found that in 
the investigated temperature range $F$ moves up with the temperature: $E_{\mathrm{C}}-F=$ $(0.76 \pm 0.01)-(5.8 \pm 0.4) \times 10^{-4} T$, where the result is in $\mathrm{eV}$ if the temperature is taken in Kelvin (Fig. 2a). For example, at $300 \mathrm{~K}$ we have $E_{\mathrm{C}}-F=0.59 \mathrm{eV}$.

\section{Discussion}

There are two possible mechanisms which can lead to the observed strong increase of the current. The first possibility is that we observe impact ionization of a deep center [4]. However, the probability of the impact ionization is proportional to the concentration of free electrons so we would expect a decrease of $E_{\text {th }}$ with an increase in temperature. Since we observe the opposite effect this indicates that we are dealing with the other mechanism, namely, with the filling of a trap with injected electrons.

The increase of $E_{\text {th }}$ with temperature is consistent with a hypothesis that the trap is located below the Fermi level [1]. Then, $E_{\text {th }}$ is proportional to the concentration $p$ of empty traps. The factor of proportionality is related to the capacitance of the sample. For non-degenerated Boltzman statistics $p$ is proportional to $\exp \left[\left(E_{\mathrm{t}}-F\right) / k T\right]$. The activation energy of $E_{\mathrm{th}}$ is equal to $-0.06 \mathrm{eV}$ (Fig. $2 \mathrm{~b}$ ). Taking into account the formula of the dependence of the Fermi level on the temperature we get $E_{\mathrm{t}}-0.76 \mathrm{eV}=-0.06 \mathrm{eV}$ so $E_{\mathrm{t}}=0.82 \mathrm{eV}$ below the conduction band. We see two possibilities. The first one is that injected electrons are trapped by a deep trap located $0.82 \mathrm{eV}$ below the conduction band. The other possibility takes into account the fact that the energy $0.82 \mathrm{eV}$ is equal to that of the EL2 level obtained from DLTS measurements [5] where the configurational barrier $E_{\mathrm{b}}=0.07 \mathrm{eV}$ adds to $0.75 \mathrm{eV}$ of the thermal EL2 energy. The point is, one can assume that in the presence of a high electric field of $2 \mathrm{kV} / \mathrm{cm}$ the dynamic occupancy equilibrium between the EL2 level and the conduction band is established mainly by electrons which are heated enough to pass over the $0.07 \mathrm{eV}$ barrier. In such a case we would expect that $E_{\mathrm{th}}$ is proportional to $\exp \left[\left(E_{\mathrm{t}}-F\right) / k T\right] \times \exp \left(E_{\mathrm{b}} / k T\right)$. From this formula we get $E_{\mathrm{t}}=0.75 \mathrm{eV}$ which coincides very well with the EL2 location.

\section{Acknowledgements}

The authors are indebted to Dr. E. Kamińska and Dr. A. Piotrowska for preparing the contacts to the sample and to Prof. M. Grynberg for valuable discussions.

\section{References}

[1] M.A. Lampert, P. Mark, Current Injection in Solids, Academic Press, New York 1970.

[2] For a review of injection current measurements see [1], Chapter 5.

[3] J.J. Mares, J. Kristofik, V. Smid, F. Demal, Solid-State Electron. 31, 1309 (1988). 
[4] C. Parracchini, V. Dallacasa, Solid State Commun. 69, 49 (1989).

[5] M. Kamińska, Phys. Sc. T 19, 551 (1987). 